\title{
The Early-Type Dwarf Galaxy Population of the Fornax Cluster
}

\author{
Steffen Mieske \\ ESO, Karl-Schwarzschild-Str.2, 85748 Garching bei München, Germany \\ email: smieske@eso.org
}

\begin{abstract}
AIMS: We analyse the photometric properties of the early-type Fornax cluster dwarf galaxy population $\left(M_{\mathrm{V}}>-17 \mathrm{mag}\right)$, based on a wide field imaging study of the central cluster area in $V$ and $I$ bandpasses. We used the instrument/telescope combination IMACS/Magellan at Las Campanas Observatory, providing much larger light collecting area and better image resolution than previous wide field imaging surveys.

METHODS: We create a fiducial sample of Fornax cluster dwarf ellipticals (dEs) in the following three steps: (1) To verify cluster membership, we measured $I$-band surface brightness fluctuations (SBF) distances to candidate dEs known from previous surveys; (2) We re-assessed morphological classifications for those candidate dEs that are too faint for SBF detection; and (3) We searched for new candidate dEs in the size-luminosity regime close to the resolution limit of previous surveys.

RESULTS: (1) We confirm cluster membership for 28 candidate dEs in the range $-16.6<$ $M_{\mathrm{V}}<-10.1$ mag by means of SBF measurement. We find no SBF background galaxy. (2) Of 51 further candidate dEs in the range $-13.2<M_{\mathrm{V}}<-8.6 \mathrm{mag}, 2 / 3$ are confirmed as probable cluster members by morphological re-assessment, while $1 / 3$ are re-classified as probable background objects. (3) We find 12 new dE candidates in the range $-12.3<M_{\mathrm{V}}<-8.8 \mathrm{mag}$, two of which are directly confirmed via SBF measurement. The resulting fiducial dE sample follows a well-defined surface brightness - magnitude relation, showing that Fornax dEs are about $40 \%$ larger than Local Group dEs. The sample also defines a colour-magnitude relation that appears slightly shallower than that of Local Group dEs. The early-type dwarf galaxy luminosity function in Fornax has a very flat faint end slope $\alpha \simeq-1.1 \pm 0.1$. We discuss these findings in the context of structure formation theories.

CONCLUSIONS: The SBF method is a very powerful tool to help constrain the faint end of the galaxy luminosity function in nearby galaxy clusters. For the Fornax cluster, morphological cluster memberships - if performed at sufficient resolution - are very reliable.
\end{abstract}

Keywords. galaxies: clusters: individual: Fornax cluster - galaxies: dwarf - galaxies: fundamental parameters - galaxies: luminosity function -techniques: photometric

This contribution is based on Mieske et al. (2006).

\section{Reference}

Mieske, S., Hilker, M., Infante, L., \& Mendes de Oliveira, C. 2006, A\&GA accepted, astro-ph/ 0610516. 